\title{
Detection and accurate localization of harmonic chipless tags
}

\author{
Davide Dardari
}

\begin{abstract}
We investigate the detection and localization properties of harmonic tags working at microwave frequencies. A two-tone interrogation signal and a dedicated signal processing scheme at the receiver are proposed to eliminate phase ambiguities caused by the short signal wavelength and to provide accurate distance/position estimation even in the presence of clutter and multipath. The theoretical limits on tag detection and localization accuracy are investigated starting from a concise characterization of harmonic backscattered signals. Numerical results show that accuracies in the order of centimeters are feasible within an operational range of a few meters in the RFID UHF band.
\end{abstract}

Keywords: Harmonic tag; RFID; Localization; Detection

\section{Introduction}

Radiofrequency identification (RFID) technology for use in real-time object identification has been widely adopted in several fields such as logistic, automotive, surveillance, and automation systems [1]. A RFID system consists of readers and tags applied to objects. The reader interrogates the tags via a wireless link to detect them.

When tag cost, size, and power consumption requirements become particularly stringent, passive tag solutions become particularly attractive. The identification of passive tags usually relies on backscatter modulation where the antenna reflection properties are changed according to the tag identifier, even though the tag's control logic and memory circuits have still to be energized to have the tag work properly [2]. Typically, passive RFID tags obtain the necessary power to operate from the radiofrequency (RF) signal sent by the reader.

We are assisting a growing demand of RFID systems with enhanced functionalities, including accurate localization and sensing, while maintaining the cost of the tag as low as possible [3, 4]. Localization capability of tags can be obtained through the exploitation of the received signal strength indicator (RSSI) or phase estimates [5]. The former does not offer good accuracies due to the unpredictable nature of the wireless fading phenomenon,

Correspondence: davide.dardari@unibo.it

WiLab, Department of Electrical, Electronic and Information Engineering (DEI) "Guglielmo Marconi", University of Bologna, Via Venezia 52, I-47521 Cesena, Italy whereas the latter suffers from phase ambiguity and clutter interference (i.e., the part of the interrogation signal backscattered by the surrounding environment). Other recent solutions exploit radically new technologies such as ultrawide bandwidth (UWB) [6-8].

Among different options, chipless tags represent the cheapest way to perform radio identification and sensing thanks to the possibility to adopt printable technologies with zero-power consumption. For these reasons, chipless tags are receiving particular attention, and several technologies are under investigation [9]. Most of them exploit the specific frequency or time response of the tag which acts as a linear passive device. The main issue when using linear chipless tags is their detection when operating in the presence of multipath, interference, and clutter which is superimposed to the useful backscattered signal.

An interesting alternative is given by harmonic tags that consist of nonlinear passive devices generating harmonic components when backscattering the incoming interrogation signal $[10,11]$. Typically, a harmonic tag is composed of a dipole antenna loaded with a nonlinear passive device. The reader generates an interrogation tone which is backscattered by the tag. Due to the presence of nonlinearities, the backscattered signal contains also harmonics of the fundamental interrogation frequency. Such harmonic components can be successfully exploited to enhance the detection of the tag because the clutter and the interference signals are received at the fundamental frequency which can be easily filtered out.

\section{是 Springer}


Harmonic tags have been introduced some years ago and have been applied to specific applications such as avalanche rescue systems [12], healthcare [13-15], sensing [16-19], and animal tracking [20-24]. A similar approach has been exploited in harmonic radar systems [25-27], in 1-bit frequency doubling tags [1], and for energy harvesting [28]. Due to the presence of nonlinear devices (e.g., the switch), harmonic spurious might also be present in standard ultrahigh frequency (UHF) tags even though they are considered undesirable effects to be limited [29]. On the contrary, this phenomenon could be potentially exploited to enhance the localization functionality of standard UHF tags if suitably treated according to the approach described in this paper.

Localization of harmonic tags can be obtained through RSSI measurements, as investigated in [30,31] or, more frequently, by adopting high-power marine radars using wideband impulsive or FM chirp-based interrogation signals [22]. Unfortunately, to provide a sufficient ranging accuracy, FM chirp-based interrogation signals must be wideband at the expense of a high-noise level that must be counteracted by adopting extremely high transmitter power levels $(>1 \mathrm{KW})$ and mechanical-steered highdirectional antennas. This prevents the exploitation of FM chirp-based radars for localization in multistatic configurations with fixed antennas.

For what the characterization of harmonic tags is concerned, several studies have been published. The electromagnetic characterization of antennas with nonlinear loads was initially carried out in [32]. Most of the studies are application oriented and present experimental results $[10,20-24,33]$. Unfortunately, no studies analyzing the fundamental detection capability and achievable localization accuracy using harmonic tags are available, at author's knowledge.

In this paper, a concise characterization of the signal backscattered by harmonic tags is given through the introduction of the specific harmonic radar cross section (SHRCS) concept. To overcome the typical ambiguity problem arising when estimating the distance through phase measurements at microwave frequencies, a simple two-tone interrogation signal is proposed providing a robust detection and accurate distance estimation (ranging) through a proper combination of the signals received at harmonic frequencies. Contrary to FM chirp radar and thanks to the narrowband nature of signals, the proposed signaling scheme can be successfully applied to the problem of tag localization using multiple receivers without the need to adopt mechanical-steered high-directivity antennas and highpower levels. Closed-form expressions of theoretical tag detection and localization accuracy limits are derived in a scenario considering one transmitter and multiple receivers.
Numerical results show the dependence of detection and localization performance on tag configurations and network topologies. Results demonstrate that accuracies in the order of a few centimeters are achievable within an operational range of some meters using simple sinusoidal interrogation signals with limited power.

\section{Harmonic tag}

A typical harmonic tag is composed of a dipole antenna loaded with a nonlinear device. The dipole antenna is generally taken with length $\lambda / 2$ at the fundamental frequency $f_{\mathrm{L}}$, being $\lambda$ the corresponding wavelength [22]. In its simplest form, the nonlinear device is composed of a Schottky diode which has a small built-in potential such that the low-power signal received by the tag is enough to make the diode operative. When excited with one ore more sinusoidal tones, the diode generates harmonic components that are reflected back through the antenna. Normally, only the fundamental and the second harmonic are effectively re-irradiated because of antenna inefficiency at other frequencies [33]. The presence of harmonic components in the received signal allows the receiver to easily identify the tag contribution among clutter components that are received superimposed to the signal backscattered by the tag at the fundamental frequency.

The current-voltage characteristic of the diode can be expressed as follows:

$$
i(t)=I_{s}\left(e^{\alpha v(t)}-1\right)
$$

where, in our numerical results, we will consider the following parameters related to the AVAGO HSMS - 286Y Schottky diode: $\alpha=38 \mathrm{~V}^{-1}, I_{s}=5 \cdot 10^{-8} \mathrm{~A}$, and junction capacitance $C_{j}=0.18 \mathrm{pF}$ [34].

Under small-signal hypothesis, (1) can be expanded in Taylor's series up to the second order term:

$$
i(t) \approx \alpha I_{s} v(t)+\frac{\alpha^{2} I_{s}}{2} v^{2}(t) .
$$

When excited by a sinusoidal tone $v(t)=v_{0} \cos \left(2 \pi f_{\mathrm{L}} t\right)$ at frequency $f_{\mathrm{L}}$, the corresponding current is:

$$
i(t) \approx k_{0} v_{0}^{2}+k_{1} v_{0} \cos \left(2 \pi f_{\mathrm{L}} t\right)+k_{2} v_{0}^{2} \cos \left(2 \pi 2 f_{\mathrm{L}} t\right)
$$

where $k_{0}=k_{2}=\frac{\alpha^{2} I_{s}}{4}$ and $k_{1}=\alpha I_{s}$.

From the Fourier analysis of the diode-antenna system, the equivalent circuits at the fundamental and second harmonic frequencies, $f_{\mathrm{L}}$ and $f_{\mathrm{H}}=2 f_{\mathrm{L}}$, can be derived as shown, respectively, in Fig. 1a, b. Denote with $\lambda_{\mathrm{L}}=c / f_{\mathrm{L}}$ and $\lambda_{\mathrm{H}}=c / f_{\mathrm{H}}$ the corresponding wavelengths, with $c$ as the speed of light. At the fundamental frequency (Fig. 1a), the diode is characterized by an effective resistance $Z_{1}=k_{1}^{-1}$ in parallel with the reactance $Z_{\mathrm{d}}\left(f_{\mathrm{L}}\right)=1 /\left(\mathrm{j} 2 \pi f_{\mathrm{L}} C_{j}\right) .{ }^{1}$ At the harmonic frequency $f_{\mathrm{H}}$ (Fig. 1b), the diode can be modeled with a dependent current generator $I_{2}=k_{2} V_{0}^{2}$ in parallel with the diode 


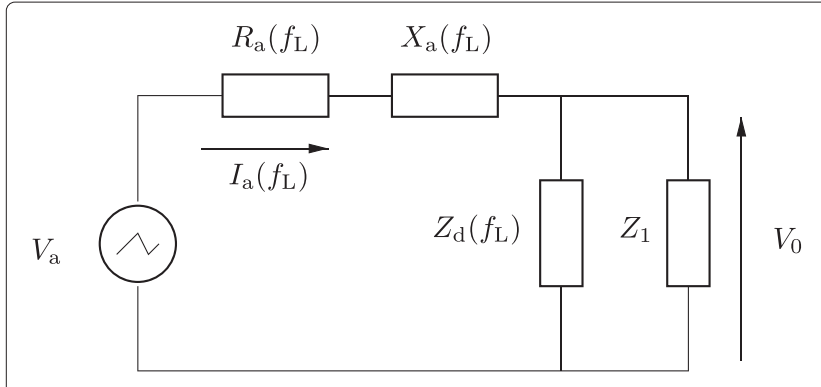

a)

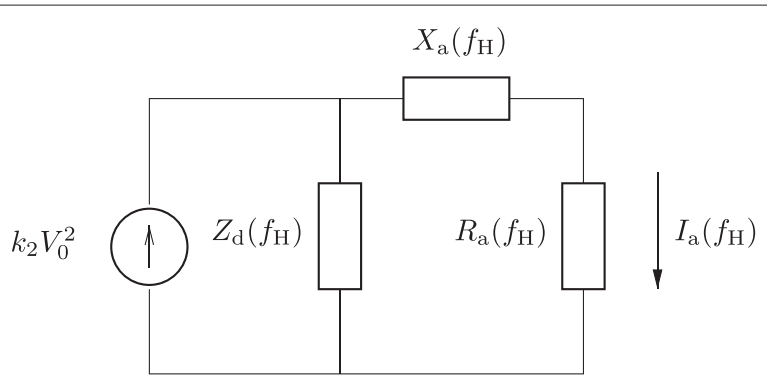

b)

Fig. 1 Harmonic tag equivalent circuits at fundamental (a) and harmonic (b) frequencies

reactance $Z_{\mathrm{d}}\left(f_{\mathrm{H}}\right)$. In Fig. $1, Z_{\mathrm{a}}(f)=R_{\mathrm{a}}(f)+{ }_{J} X_{\mathrm{a}}(f)$ denotes the antenna impedance at frequency $f$ and $V_{\mathrm{a}}$ the (e.m.f.) induced in the antenna by the effective impinging electric field $E_{\text {inc }}$ given by:

$$
V_{\mathrm{a}}=l_{0} E_{\mathrm{inc}}=\lambda_{\mathrm{L}} \sqrt{\frac{G_{\mathrm{t}}\left(f_{\mathrm{L}}\right) R_{\mathrm{a}}\left(f_{\mathrm{L}}\right) W_{\mathrm{inc}}}{\pi}}
$$

where $W_{\text {inc }}$ is the power density incident to the tag at the fundamental frequency and $l_{0}$ and $G_{\mathrm{t}}\left(f_{\mathrm{L}}\right)$ are, respectively, the antenna electric length and gain at $f=f_{\mathrm{L}}$.

According to the equivalent circuits in Fig. 1 and classical antenna theory results [35], the effective isotropic radiated power (EIRP) of the backscattered components at the fundamental and harmonic frequencies are, respectively, $P_{\mathrm{sc}}\left(f_{\mathrm{L}}\right)=\left|I_{a}\left(f_{\mathrm{L}}\right)\right|^{2} R_{a}\left(f_{\mathrm{L}}\right)$ and $P_{\mathrm{sc}}\left(f_{\mathrm{H}}\right)=\left|I_{a}\left(f_{\mathrm{H}}\right)\right|^{2} R_{a}\left(f_{\mathrm{H}}\right)$, with:

$$
\begin{aligned}
& I_{\mathrm{a}}\left(f_{\mathrm{L}}\right)=\frac{V_{\mathrm{a}}}{Z_{\mathrm{a}}\left(f_{\mathrm{L}}\right)+Z_{1} \| Z_{\mathrm{d}}\left(f_{\mathrm{L}}\right)} \\
& I_{\mathrm{a}}\left(f_{\mathrm{H}}\right)=\frac{k_{2} V_{0}^{2}\left(Z_{\mathrm{d}}\left(f_{\mathrm{H}}\right) \| Z_{\mathrm{a}}\left(f_{\mathrm{H}}\right)\right)}{Z_{\mathrm{a}}\left(f_{\mathrm{H}}\right)}
\end{aligned}
$$

and

$$
V_{0}=\frac{V_{\mathrm{a}}\left(Z_{\mathrm{d}}\left(f_{\mathrm{L}}\right) \| Z_{1}\right)}{Z_{\mathrm{d}}\left(f_{\mathrm{L}}\right) \| Z_{1}+Z_{\mathrm{a}}\left(f_{\mathrm{L}}\right)} .
$$

From simple circuit analysis, it can be easily shown that $P_{\text {sc }}\left(f_{\mathrm{L}}\right)=W_{\text {inc }} \sigma_{\mathrm{L}}$, where:

$$
\sigma_{\mathrm{L}}=\frac{\lambda_{\mathrm{L}}^{2} G_{\mathrm{t}}\left(f_{\mathrm{L}}\right) R_{\mathrm{a}}^{2}\left(f_{\mathrm{L}}\right)}{\pi\left(Z_{\mathrm{a}}\left(f_{\mathrm{L}}\right)+Z_{1} \| Z_{\mathrm{d}}\left(f_{\mathrm{L}}\right)\right)^{2}}
$$

is the radar cross-section (RCS) of the tag at the fundamental frequency. ${ }^{2}$ In case of network matching, it is $\sigma_{\mathrm{L}}=\frac{\lambda_{\mathrm{L}}^{2} G_{\mathrm{t}}\left(f_{\mathrm{L}}\right)}{4 \pi}$, which is the antenna effective area.

By analyzing the equivalent circuit in Fig. 1b, it turns out that $P_{\mathrm{sc}}\left(f_{\mathrm{H}}\right)$ is proportional to $W_{\text {inc }}^{2}$, that is, $P_{\mathrm{sc}}\left(f_{\mathrm{H}}\right)=$ $\sigma_{\mathrm{s}} W_{\text {inc }}^{2}$, where:

$$
\begin{aligned}
\sigma_{\mathrm{s}}= & k_{2}^{2} R_{a}\left(f_{\mathrm{H}}\right) G_{\mathrm{t}}\left(f_{\mathrm{L}}\right)^{2} R_{a}^{2}\left(f_{\mathrm{L}}\right) \frac{\lambda_{\mathrm{L}}^{4}}{\pi^{2}} \\
& \cdot\left|\frac{\left(Z_{d}\left(f_{\mathrm{H}}\right) \| Z_{a}\left(f_{\mathrm{H}}\right)\right.}{Z_{a}\left(f_{\mathrm{H}}\right)}\right|^{2}\left|\frac{Z_{d}\left(f_{\mathrm{L}}\right)|| Z_{1}}{Z_{d}\left(f_{\mathrm{L}}\right)|| Z_{1}+Z_{\mathrm{a}}\left(f_{\mathrm{L}}\right)}\right|^{4} .
\end{aligned}
$$

Define the conversion RCS (or harmonic RCS):

$$
\sigma_{\mathrm{h}}\left(W_{\text {inc }}\right)=P_{\mathrm{sc}}\left(f_{\mathrm{H}}\right) / W_{\text {inc }}=\sigma_{\mathrm{s}} \cdot W_{\text {inc }}
$$

which is not constant but depends linearly on the incident power density $W_{\text {inc. This }}$ relationship is not accounted for in $[22,23]$, where $\sigma_{\mathrm{h}}$ is considered as constant, and it is conjectured empirically in [33]. Here we have shown that this linear dependence comes from the secondorder Taylor series expansion approximation. Parameter $\sigma_{\mathrm{s}}\left[\mathrm{m}^{4} / \mathrm{W}\right]$ represents the harmonic RCS coefficient per incident power density unit, namely the specific harmonic radar cross section (SHRCS), and accounts for the no perfect matching between the diode and the tag as well as the diode efficiency. As a consequence, the tag conversion gain depends on $W_{\text {inc }}$ as well and results:

$$
G\left(W_{\text {inc }}\right)=\frac{4 \pi}{\lambda_{\mathrm{H}}^{2}} \sigma_{\mathrm{s}} W_{\mathrm{inc}}=W_{\mathrm{inc}} G_{\mathrm{t}}\left(f_{\mathrm{H}}\right) G_{\mathrm{t}}\left(f_{\mathrm{L}}\right)^{2} \eta_{T} \frac{\lambda_{\mathrm{L}}^{4}}{4 \pi \lambda_{\mathrm{H}}^{2}}
$$

where $\eta_{T}$ is the tag efficiency in [ $\mathrm{W}^{-1}$ ] as defined in [21].

The model in (9) and (10) is valid under the smallsignal approximation (3). In practice, it provides accurate results for low values of the incident power density $W_{\text {inc }}$, i.e., long distances, whereas it leads to a conservative performance evaluation for large values of $W_{\text {inc. }}$. This is not a drawback as the subsequent analysis is addressed to establish the maximum operating range of the system for which the small-signal approximation is satisfactory.

The result in (9) provides an easy tool to optimize the antenna configuration in terms of matching network, antenna length $L$, and feeding point position. As an example, in Fig. 2, the SHRCS $\sigma_{\mathrm{s}}$ as a function of the normalized antenna length $L / \lambda_{\mathrm{L}}$ is shown for the AVAGO 


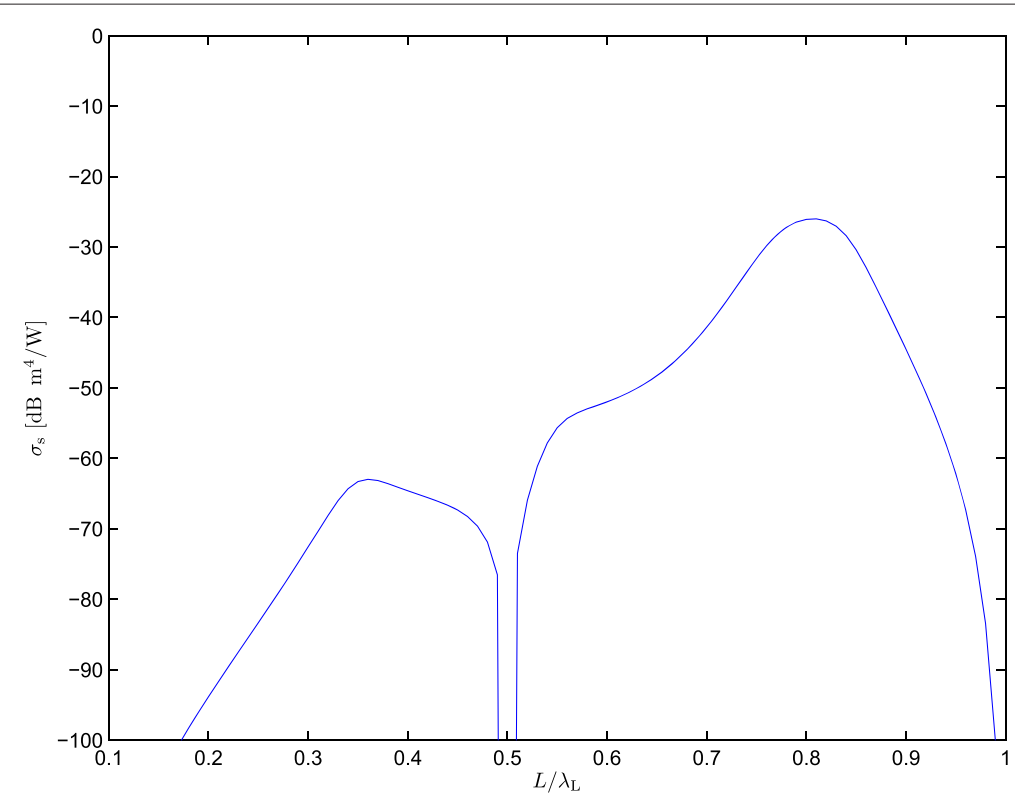

Fig. $2 \mathrm{SHRCS} \sigma_{\mathrm{S}}\left[\mathrm{dB} \mathrm{m} \mathrm{m}^{4} / \mathrm{W}\right]$ as a function of the normalized antenna length $L / \lambda_{L}$. A 0.5-mm dipole wire diameter is considered

HSMS - 286Y diode considered using theoretical expressions for what the evaluation of $Z_{\mathrm{a}}(f)$ is concerned [35]. As can be noticed, the optimum dipole length is $L=4 \lambda_{\mathrm{L}} / 5$ to which corresponds SHRCS $\sigma_{\mathrm{s}}=-26\left[\mathrm{~dB} \mathrm{~m}^{4} / \mathrm{W}\right]$. An extensive analysis of other configurations is out of the scope of this paper. Some examples can be found in $[21,22,33]$. In general, once one has characterized the SHRCS via simulation or experimentally, its value represents an input for the following theoretical framework on the detection and localization capabilities of harmonic tags.

\section{Tag detection and ranging}

\section{The proposed system}

With reference to the scheme reported in Fig. 3, consider a transmitter that emits an interrogation signal, a harmonic tag located at $d_{\mathrm{T}}$ meters from the transmitter, and one receiver placed at $d_{\mathrm{R}}$ meters from the tag. When the transmitter and receiver are co-located, it is $d_{\mathrm{T}}=d_{\mathrm{R}}$. We propose a transmitted interrogation signal composed of two tones at slightly different frequencies $f_{1}$ and $f_{2}$ :

$$
s(t)=\sqrt{2 P_{\mathrm{t}} R} \cos \left(2 \pi f_{1} t+\theta_{1}\right)+\sqrt{2 P_{\mathrm{t}} R} \cos \left(2 \pi f_{2} t+\theta_{2}\right)
$$

where $P_{\mathrm{t}}$ is the transmitted power associated to each tone, $R$ is the matching resistance, and $f_{2}=f_{1}+\Delta f$ with $\Delta f \ll f_{1}, f_{2}$. For instance, $f_{1}$ and $f_{2}$ could belong to the UHF RFID band 865-870 Mhz with $\Delta f$ in the order of a few MHz. In general, the phase difference $\theta_{2}-\theta_{1}$ between the two tones is not know if independent oscillators are used to generate them. On the contrary, $\theta_{2}-\theta_{1}=0$ if the tones are generated coherently. ${ }^{3}$ Hereafter, all frequencies around $f_{\mathrm{L}} \triangleq f_{1} \approx f_{2}$ are referred to as fundamental (interrogation frequency), whereas all frequencies around $f_{\mathrm{H}} \triangleq 2 f_{1} \approx 2 f_{2} \approx f_{1}+f_{2}$ are referred to as harmonics (response frequency).

We indicate with $G_{\mathrm{tx}}\left(f_{\mathrm{L}}\right)$ and $G_{\mathrm{rx}}\left(f_{\mathrm{H}}\right)$, respectively, the transmitter and receiver antenna gains. Note that $G_{\mathrm{rx}}\left(f_{\mathrm{H}}\right)$ refers to the receiver antenna gain at the harmonic frequency $f_{\mathrm{H}}$, whereas $G_{\mathrm{tx}}\left(f_{\mathrm{L}}\right)$ refers to the transmitter antenna gain at the fundamental frequency $f_{\mathrm{L}}$. We consider the antenna characteristics constant within a bandwidth $\Delta f$.

Denote with $H_{\mathrm{T}}(f)$ and $H_{\mathrm{R}}(f)$ the channel transfer functions of the transmitter-tag link and tagreceiver link, respectively. For further convenience, define $\alpha_{\mathrm{T}} \triangleq\left|H_{\mathrm{T}}\left(f_{1}\right)\right| \approx\left|H_{\mathrm{T}}\left(f_{2}\right)\right|, \theta_{\mathrm{T}}(f) \triangleq \arg H_{\mathrm{T}}(f)$, $\alpha_{\mathrm{R}} \triangleq\left|H_{\mathrm{R}}\left(2 f_{1}\right)\right| \approx\left|H_{\mathrm{T}}\left(2 f_{2}\right)\right|$, and $\theta_{\mathrm{R}}(f) \triangleq \arg H_{\mathrm{R}}(f)$.

The electric field at the tag is:

$$
\begin{aligned}
e_{\mathrm{inc}}(t)= & \frac{E_{\mathrm{inc}}}{\sqrt{2}} \cos \left(2 \pi f_{1}\left(t-\tau_{\mathrm{T}}\right)+\theta_{1}+\theta_{\mathrm{T}}\left(f_{1}\right)\right) \\
& +\frac{E_{\mathrm{inc}}}{\sqrt{2}} \cos \left(2 \pi f_{2}\left(t-\tau_{\mathrm{T}}\right)+\theta_{2}+\theta_{\mathrm{T}}\left(f_{2}\right)\right)
\end{aligned}
$$

where the effective electric field amplitude is given by:

$$
E_{\mathrm{inc}}=\sqrt{\frac{P_{\mathrm{t}} G_{\mathrm{tx}}\left(f_{\mathrm{L}}\right) \eta_{0} \alpha_{\mathrm{T}}^{2}}{4 \pi d_{\mathrm{T}}^{2}}}=\sqrt{W_{\mathrm{inc}} \eta_{0} \alpha_{\mathrm{T}}^{2}}
$$

with $\eta_{0}=377 \mathrm{Ohm}$, and $\tau_{\mathrm{T}}=d_{\mathrm{T}} / c$ the signal time-offlight (TOF). 


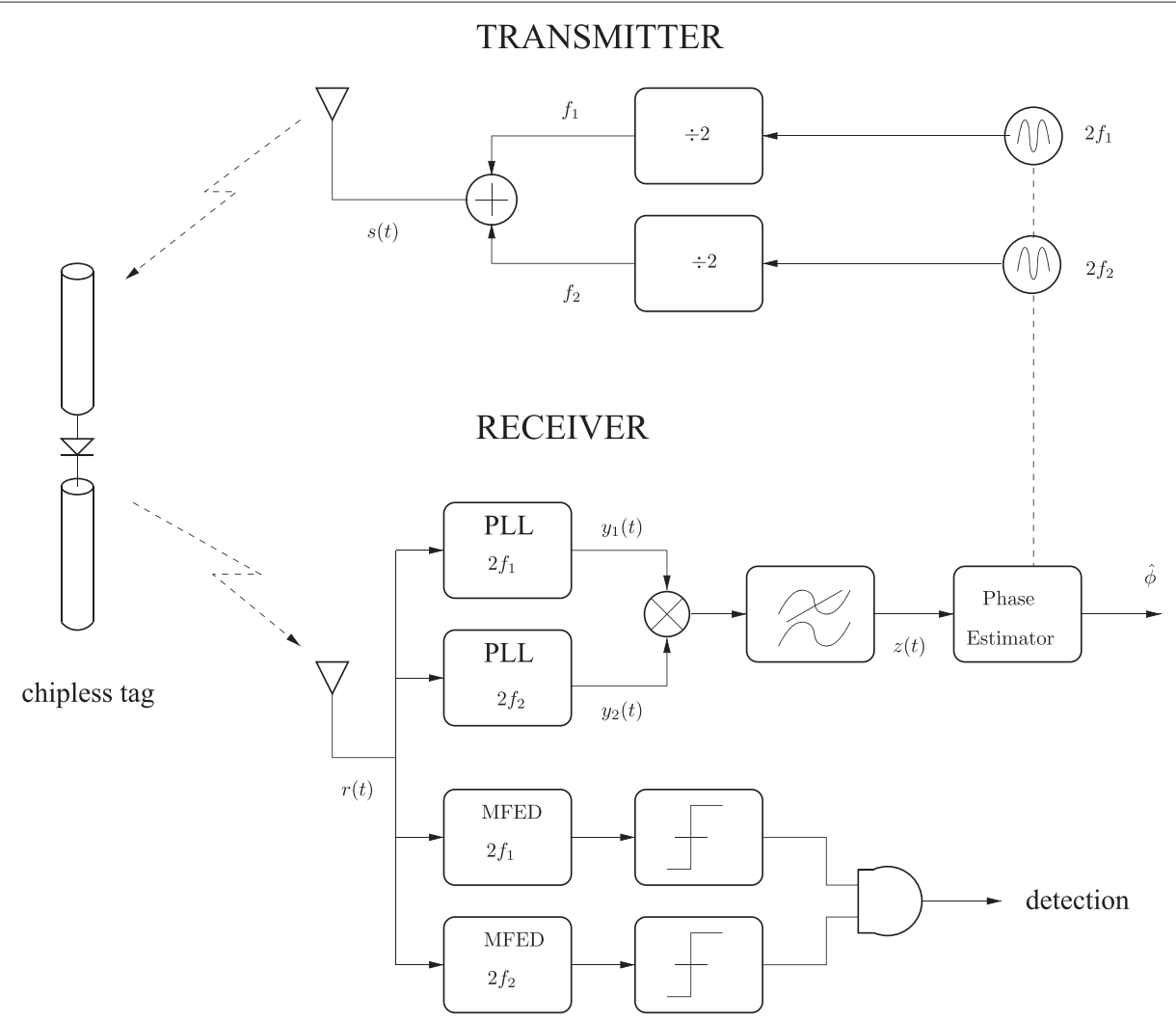

Fig. 3 The proposed scheme for tag detection and ranging

According to (3), the harmonic components depend on $e_{\text {inc }}^{2}(t)$ which can be expanded as:

$$
\begin{gathered}
e_{\mathrm{inc}}^{2}(t)=\frac{E_{\mathrm{inc}}^{2}}{2}+\frac{E_{\mathrm{inc}}^{2}}{4} \cos \left(2 \pi 2 f_{1}\left(t-\tau_{\mathrm{T}}\right)+2 \theta_{1}+2 \theta_{\mathrm{T}}\left(f_{1}\right)\right) \\
+\frac{E_{\mathrm{inc}}^{2}}{4} \cos \left(2 \pi 2 f_{2}\left(t-\tau_{\mathrm{T}}\right)+2 \theta_{2}+2 \theta_{\mathrm{T}}\left(f_{2}\right)\right) \\
+\frac{E_{\mathrm{inc}}^{2}}{2} \cos \left(2 \pi 2\left(f_{1}+f_{2}\right)\left(t-\tau_{\mathrm{T}}\right)+\theta_{1}+\theta_{2}\right. \\
\left.\quad+\theta_{\mathrm{T}}\left(f_{1}\right)+\theta_{\mathrm{T}}\left(f_{2}\right)\right) \\
+\frac{E_{\mathrm{inc}}^{2}}{2} \cos \left(2 \pi 2\left(f_{2}-f_{1}\right)\left(t-\tau_{\mathrm{T}}\right)+\theta_{1}+\theta_{2}\right. \\
\left.\quad+\theta_{\mathrm{T}}\left(f_{2}\right)-\theta_{\mathrm{T}}\left(f_{1}\right)\right) .
\end{gathered}
$$

From (15), it is evident that the second harmonic contributions to the backscattered signal contain the frequencies $0,2 f_{1}, 2 f_{2}, f_{1}+f_{2}$, and $f_{2}-f_{1}=\Delta f$, of which the DC and $f_{2}-f_{1}$ components are filtered out by the irradiating element and hence neglected in the following.

As a consequence, we concentrate our attention to the components of interest at harmonic frequencies $2 f_{1}, 2 f_{2}$, and $f_{1}+f_{2}$ and at fundamental frequencies $f_{1}$ and $f_{2}$.

The characterization of the tag response in the presence of one exciting tone carried out in the previous section can still be applied to calculate the backscattered power at frequencies $f_{1}, f_{2}, 2 f_{1}$, and $2 f_{2}$ in the presence of two exciting tones at frequencies $f_{1}$ and $f_{2}$ thanks to the small-signal approximation. This does not hold for the calculation of the harmonic at frequency $f_{1}+f_{2}$, which, however, is out of interest for our application, as will be clearer later.

The received power of the fundamental backscattered components at $f_{1}$ and $f_{2}$ is:

$$
P_{\mathrm{r}}\left(f_{\mathrm{L}}\right)=\frac{P_{\mathrm{t}} G_{\mathrm{tx}}\left(f_{\mathrm{L}}\right) G_{\mathrm{rx}}\left(f_{\mathrm{H}}\right) \lambda_{\mathrm{L}}^{2} \sigma_{\mathrm{L}} \alpha_{\mathrm{T}}^{2} \alpha_{\mathrm{R}}^{2}}{(4 \pi)^{3} d_{\mathrm{T}}^{2} d_{\mathrm{R}}^{2}}
$$

which is the well-known radar equation when $d_{\mathrm{T}}=d_{\mathrm{R}}$ and $\alpha_{\mathrm{T}}=\alpha_{\mathrm{R}}=1$ (i.e., free-space condition).

The received power of the harmonic components at $2 f_{1}$ and $2 f_{2}$ is:

$$
\begin{aligned}
P_{\mathrm{r}}\left(f_{\mathrm{H}}\right) & =\frac{P_{\mathrm{t}} G_{\mathrm{tx}}\left(f_{\mathrm{L}}\right) G_{\mathrm{rx}}\left(f_{\mathrm{H}}\right) \lambda_{\mathrm{H}}^{2} \sigma_{\mathrm{s}} \alpha_{\mathrm{T}}^{2} \alpha_{\mathrm{R}}^{2}}{(4 \pi)^{3} d_{\mathrm{T}}^{2} d_{\mathrm{R}}^{2}} \cdot \frac{P_{\mathrm{t}} G_{\mathrm{tx}}\left(f_{\mathrm{L}}\right) \alpha_{\mathrm{T}}^{2}}{4 \pi d_{\mathrm{T}}^{2}} \\
& =\frac{\left(P_{\mathrm{t}} G_{\mathrm{tx}}\left(f_{\mathrm{L}}\right) \lambda_{\mathrm{H}}\right)^{2} G_{\mathrm{rx}}\left(f_{\mathrm{H}}\right) \sigma_{\mathrm{s}} \alpha_{\mathrm{T}}^{4} \alpha_{\mathrm{R}}^{2}}{(4 \pi)^{4} d_{\mathrm{R}}^{2} d_{\mathrm{T}}^{4}} \\
& =P_{\mathrm{r} 0}\left(f_{\mathrm{H}}\right) d_{\mathrm{R}}^{-2} d_{\mathrm{T}}^{-4}
\end{aligned}
$$


having defined:

$$
P_{\mathrm{r} 0}\left(f_{\mathrm{H}}\right)=\frac{\operatorname{EIRP}^{2} \lambda_{\mathrm{H}}^{2} G_{\mathrm{rx}}\left(f_{\mathrm{H}}\right) \sigma_{\mathrm{s}} \alpha_{\mathrm{T}}^{4} \alpha_{\mathrm{R}}^{2}}{(4 \pi)^{4}}
$$

and EIRP $=P_{\mathrm{t}} G_{\mathrm{tx}}\left(f_{\mathrm{L}}\right)$. As can be noticed, the received power decreases with $d_{\mathrm{T}}^{4} d_{\mathrm{R}}^{2}$. This large attenuation with the distance is in part recovered by the square in the EIRP term in (18).

Therefore, the signal backscattered by the harmonic tag seen by the receiver is:

$$
\begin{aligned}
r(t)= & \sqrt{2 P_{\mathrm{r}}\left(f_{\mathrm{L}}\right) R} \cos \left(2 \pi f_{1}(t-\tau)+\theta_{1}+\theta_{\mathrm{T}}\left(f_{1}\right)\right) \\
& +\sqrt{2 P_{\mathrm{r}}\left(f_{\mathrm{L}}\right) R} \cos \left(2 \pi f_{2}(t-\tau)+\theta_{2}+\theta_{\mathrm{T}}\left(f_{2}\right)\right) \\
& +\sqrt{2 P_{\mathrm{r}}\left(f_{\mathrm{H}}\right) R} \cos \left(2 \pi 2 f_{1}(t-\tau)+2 \theta_{1}+2 \theta_{\mathrm{T}}\left(f_{1}\right)\right. \\
& \left.+\theta_{\mathrm{R}}\left(2 f_{1}\right)\right)+\sqrt{2 P_{\mathrm{r}}\left(f_{\mathrm{H}}\right) R} \cos \left(2 \pi 2 f_{2}(t-\tau)\right. \\
& \left.+2 \theta_{2}+2 \theta_{\mathrm{T}}\left(f_{2}\right)+\theta_{\mathrm{R}}\left(2 f_{2}\right)\right) \\
& +\sqrt{2\left(P_{\mathrm{r}}\left(f_{1}+f_{2}\right)\right) R} \cos \left(2 \pi\left(f_{1}+f_{2}\right)(t-\tau)+\theta_{1}\right. \\
& \left.+\theta_{2}+\theta_{\mathrm{T}}\left(f_{1}\right)+\theta_{\mathrm{T}}\left(f_{2}\right)+\theta_{\mathrm{R}}\left(f_{1}+f_{2}\right)\right)+c(t)+n(t),
\end{aligned}
$$

in which $n(t)$ is the additive white Gaussian noise (AWGN) with one-side power spectral density $N_{0}, c(t)$ is the clutter component caused by the reflection of the interrogation signal by the surrounding environment at fundamental frequencies, and $\tau=\tau_{\mathrm{T}}+\tau_{\mathrm{R}}$, where $\tau_{\mathrm{T}}=d_{\mathrm{T}} / c$ and $\tau_{\mathrm{R}}=d_{\mathrm{R}} / c$ are, respectively, the transmitter-tag and the tag-receiver TOFs. The clutter can be eliminated by considering only the backscattered components at harmonic frequencies. This is the main advantage of harmonic tags as already anticipated in the "Introduction" section.

\section{Tag detection}

To detect the tag, it is sufficient to analyze the presence of the harmonic sinusoidal components at $2 f_{1}$ and $2 f_{2}$. The detection of sinusoidal signals in noise with a random unknown phase is a well-known problem in detection theory which is solved optimally by employing matched filter-envelope detectors (MFEDs) (also called quadrature matched filter) [36]. As shown in Fig. 3, two MFEDs followed by a comparator with threshold $\gamma$ are considered to detect the two tones at frequencies $2 f_{1}$ and $2 f_{2}$ embedded in the received signal $r(t)$. We adopt the Neyman-Pearson criterium to set the threshold $\gamma$ starting from a target probability of false alarm $P_{\mathrm{F}}$ whose value is applicationdependent.

Due to the symmetry of the problem, the probability of a false alarm of the two detectors is the same and it is given by [36]:

$$
P_{\mathrm{F}_{1}}=P_{\mathrm{F}_{2}}=\exp \left\{-\frac{\gamma}{N_{0}}\right\} \text {. }
$$

To make the global detector more robust to false alarms, we employ the strategy according to which the tag is considered detected only if both detectors detect it. Since thermal noise components at different frequencies are independent, the global probability of a false alarm is $P_{\mathrm{F}}=P_{\mathrm{F}_{1}}^{2}=P_{\mathrm{F}_{2}}^{2}$.

The probability of detection of each detector is [36]:

$$
P_{\mathrm{D}_{1}}=P_{\mathrm{D}_{2}}=Q\left(\sqrt{a}, \sqrt{\frac{2 \gamma}{N_{0}}}\right)
$$

being $a=2 P_{\mathrm{r}}\left(f_{\mathrm{H}}\right) T / N_{0}$ the signal-to-noise ratio (SNR), $T$ the observation time, and $Q(\cdot, \cdot)$ the Marcum's $Q$-function.

In virtue of the independency of the two detectors, the global probability of detection $P_{\mathrm{D}}$ is:

$$
\begin{aligned}
P_{\mathrm{D}} & =1-\left(1-P_{\mathrm{D}_{1}}\right)\left(1-P_{\mathrm{D}_{2}}\right) \\
& =2 Q\left(\sqrt{a}, \sqrt{-\ln P_{\mathrm{F}}}\right)-Q^{2}\left(\sqrt{a}, \sqrt{-\ln P_{\mathrm{F}}}\right) .
\end{aligned}
$$

\section{Range estimate}

To estimate the total distance $d=d_{\mathrm{T}}+d_{\mathrm{R}}$ without ambiguities caused by phase periodicity, we propose the scheme shown in Fig. 3 where the received signal $r(t)$ is processed by two phase-locked loop (PLL) filters tuned, respectively, at $2 f_{1}$ and $2 f_{2}$, characterized by a noise equivalent bandwidth $B_{\text {eq }}$. At the output of each PLL, we obtain the signals:

$y_{1}(t)=\cos \left(2 \pi 2 f_{1}(t-\tau)+2 \theta_{1}+2 \theta_{\mathrm{T}}\left(f_{1}\right)+\theta_{\mathrm{R}}\left(2 f_{1}\right)+w_{1}\right)$

$y_{2}(t)=\cos \left(2 \pi 2 f_{2}(t-\tau)+2 \theta_{2}+2 \theta_{\mathrm{T}}\left(f_{2}\right)+\theta_{\mathrm{R}}\left(2 f_{2}\right)+w_{2}\right)$

where $w_{1}$ and $w_{1}$ are the phase noise residuals characterized by a power $N_{\mathrm{w}}=\mathbb{E}\left[w_{1}^{2}\right]=\mathbb{E}\left[w_{2}^{2}\right]=\frac{N_{0} B_{\mathrm{eq}}}{2 P_{\mathrm{r}}\left(f_{\mathrm{H}}\right)}$, we approximate as Gaussian random variables (RVs) [37].

According to the scheme proposed in Fig. 3, signals $y_{1}(t)$ and $y_{2}(t)$ are multiplied by each other and filtered by a low-pass filter thus obtaining:

$$
\begin{array}{r}
z(t)=\cos \left(4 \pi \Delta f t-4 \pi \Delta f \tau+2\left(\theta_{2}-\theta_{1}\right)+2\left(\theta_{\mathrm{T}}\left(f_{2}\right)\right.\right. \\
\left.\left.-\theta_{\mathrm{T}}\left(f_{1}\right)\right)+\theta_{\mathrm{R}}\left(2 f_{2}\right)-\theta_{\mathrm{R}}\left(2 f_{1}\right)+w_{2}-w_{1}\right) .
\end{array}
$$

If $\Delta f$ is much less than the channel coherence bandwidth, as typically happens in line-of-sight (LOS) conditions, the differences $2 \theta_{\mathrm{T}}\left(f_{1}\right)-2 \theta_{\mathrm{T}}\left(f_{2}\right)$ and $\theta_{\mathrm{R}}\left(2 f_{1}\right)-\theta_{\mathrm{R}}\left(2 f_{2}\right)$ are expected to be small in general [38]. Moreover, the phase difference $\theta_{2}-\theta_{1}$ is zero if the tones are coherently generated at the transmitter or can be removed if the transmitter and receivers are synchronized. Under these conditions, we will not consider these terms in the remainder. 
The phase estimate of the tone in (25) is:

$$
\hat{\phi}=-4 \pi \tau \Delta f+u
$$

being $u \triangleq w_{2}-w_{1}$. From (26), an estimate $\hat{\tau}=\frac{\hat{\phi}}{4 \pi \Delta f}$ of the TOF $\tau$, and hence of the total distance $d$, can be obtained. Note that the adoption of two interrogation tones allows the operation in (25) and (26) from which, due to the periodicity of $2 \pi$ of the phase estimate, no ambiguities in ranging arise for distances less than $d_{\max }=\frac{c}{4 \Delta f}$. Since $\Delta f$ is in the order of a few $\mathrm{MHz}, d_{\max }$ takes values that are of interest for most applications. For example, for $\Delta f=4 \mathrm{MHz}$, it is $d_{\max } \approx 18 \mathrm{~m}$. On the contrary, in the presence of only one interrogation tone, the distance would have been estimated directly from the phase of the sinusoid in (23) from which $d$ is obtained with an ambiguity of $d_{\max }=\frac{c}{4 f_{1}} \approx 9 \mathrm{~cm}$, which is obviously too small for any practical application of the system.

The range estimate is simply obtained by:

$$
r=\hat{\tau} c=\frac{\hat{\phi} c}{4 \pi \Delta f}=d+\epsilon
$$

where $\epsilon=-\frac{u c}{4 \pi \Delta f}$ represents the Gaussian ranging error with mean $\mathbb{E}[\epsilon]=0$ and variance $\sigma_{\mathrm{r}}^{2}=\mathbb{E}\left[\epsilon^{2}\right]=\frac{2 N_{\mathrm{w}} c^{2}}{(4 \pi \Delta f)^{2}}=$ $\frac{N_{0} B_{\mathrm{eq}}}{P_{\mathrm{r}}\left(f_{\mathrm{H}}\right)(4 \pi \Delta f)^{2}} \cdot{ }^{4}$

\section{Extension to multiple receivers for localization}

So far, the system illustrated in Fig. 3 allows estimating the total transmitter-tag-receiver distance. In this section, we extend our analysis considering a network composed of one transmitter, located at coordinates $\left(x_{\mathrm{T}}, y_{\mathrm{T}}\right)$ and emitting the interrogation signal defined in the previous section, one harmonic tag at position $\mathbf{p}=(x, y)$, and $N_{\mathrm{r}}$ receivers deployed at coordinates $\left(x_{\mathrm{R} i}, y_{\mathrm{R} i}\right)$, with $i=1,2, \ldots, N_{\mathrm{r}}$, as depicted in Fig. 4 (multistatic configuration). The purpose of the network is to determine the position of the tag starting from the set $\mathbf{r}=$ $\left[r_{1}, r_{2}, \ldots, r_{N_{\mathrm{r}}}\right]$ of range estimates $r_{i}=d_{\mathrm{T}}+d_{\mathrm{R} i}+\epsilon_{i}$ calculated by the $N_{\mathrm{r}}$ receivers, having denoted with $d_{\mathrm{T}}$ the distance between the transmitter and the tag and $d_{\mathrm{R} i}$ the distance between the tag and the $i$ th receiver. According to (17) and (27), $\epsilon_{i}$ represents the range estimation error which is Gaussian distributed with a zero mean and variance $\mathbb{E}\left[\epsilon_{i}^{2}\right]=\sigma_{0}^{2} d_{\mathrm{T}}^{\alpha} d_{\mathrm{R} i}^{\beta}$, being $\alpha=4, \beta=2$, and $\sigma_{0}^{2}=\frac{c^{2} N_{0} B_{\mathrm{eq}}}{P_{\mathrm{r} 0}\left(f_{\mathrm{H}}\right)(4 \pi \Delta f)^{2}}$ the estimation error variance when $d_{\mathrm{T}}=d_{\mathrm{R} i}=1 \mathrm{~m}$.

The theoretical limit on the mean square error (MSE) of any position estimator $\widehat{\mathbf{p}}=(\widehat{x}, \widehat{y})$ of $\mathbf{p}$, the position error bound (PEB), exploiting $N_{\mathrm{r}}$ range observations can be derived from the Cramer-Rao bound ${ }^{5}$ [36]

$$
\mathbb{E}_{\mathbf{r}}\left\{(\mathbf{p}-\widehat{\mathbf{p}})(\mathbf{p}-\widehat{\mathbf{p}})^{\mathrm{T}}\right\} \succeq \mathbf{J}^{-1}(\mathbf{p})
$$

where $\mathbb{E}_{\mathbf{r}}\{\cdot\}$ is the statistical expectation with respect to the random vector $\mathbf{r}$ and $\mathbf{J}(\mathbf{p})$ is the Fisher information matrix (FIM) given by:

$$
\mathbf{J}(\mathbf{p})=\mathbb{E}_{\mathbf{r}}\left\{\left[\nabla_{\mathbf{p}} \ln (f(\mathbf{r} \mid \mathbf{p}))\right]\left[\nabla_{\mathbf{p}} \ln (f(\mathbf{r} \mid \mathbf{p}))\right]^{\mathrm{T}}\right\}
$$

having indicated with $f(\mathbf{r} \mid \mathbf{p})$ the probability density function (p.d.f.) of the observation vector $\mathbf{r}$ conditioned on $\mathbf{p}$. The PEB is then defined as [39]:

$$
\operatorname{PEB}(\mathbf{p}) \triangleq \sqrt{\operatorname{tr}\left\{\mathbf{J}^{-1}(\mathbf{p})\right\}}
$$

where $\operatorname{tr}\{\cdot\}$ is the trace of a square matrix. Considering independent observations, we have:

$$
f(\mathbf{r} \mid \mathbf{p})=\prod_{i=1}^{N_{\mathrm{r}}} f_{i}\left(r_{i} \mid \mathbf{p}\right)
$$

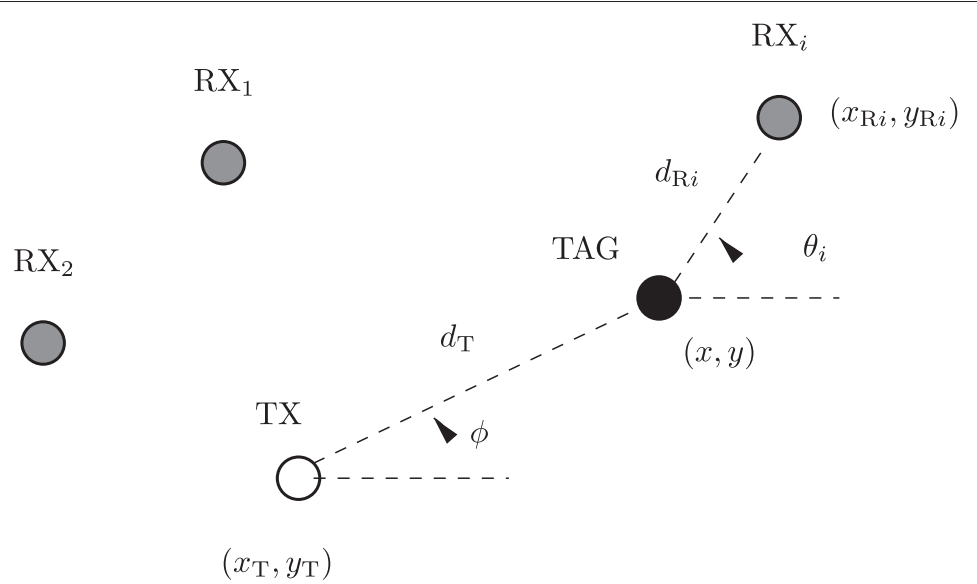

$\mathrm{RX}_{N_{\mathrm{r}}}$

Fig. 4 Considered layout for the localization of the tag using multiple receivers 
where $f_{i}\left(r_{i} \mid \mathbf{p}\right)$ is the p.d.f. of the $i$ th observation conditioned on $\mathbf{p}$ given $\mathrm{by}^{6}$ :

$$
f_{i}\left(r_{i} \mid \mathbf{p}\right)=\frac{1}{\sqrt{2 \pi \sigma_{0}^{2} d_{\mathrm{T}}^{\alpha} d_{\mathrm{R} i}^{\beta}}} \exp \left\{-\frac{\left(r_{i}-d_{\mathrm{T}}-d_{\mathrm{R} i}\right)^{2}}{2 \sigma_{0}^{2} d_{\mathrm{T}}^{\alpha} d_{\mathrm{R} i}^{\beta}}\right\} .
$$

Considering that:

$$
\nabla_{\mathbf{p}} \ln (f(\mathbf{r} \mid \mathbf{p}))=\sum_{i=1}^{N_{\mathrm{r}}} \frac{1}{f_{i}\left(r_{i} \mid \mathbf{p}\right)}\left[\begin{array}{c}
\frac{\partial f_{i}\left(r_{i} \mid \mathbf{p}\right)}{\partial x} \\
\frac{\partial f_{i}\left(r_{i} \mid \mathbf{p}\right)}{\partial y}
\end{array}\right]
$$

we obtain:

$$
\begin{aligned}
\mathbf{J}(\mathbf{p})=\mathbb{E}_{\mathbf{r}} & \left\{\sum_{i=1}^{N_{\mathrm{r}}} \sum_{j=1}^{N_{\mathrm{r}}} \frac{1}{f_{i}\left(r_{i} \mid \mathbf{p}\right)} \frac{1}{f_{j}\left(r_{j} \mid \mathbf{p}\right)}\right. \\
& \left.\times\left[\begin{array}{c}
\frac{\partial f_{i}\left(r_{i} \mid \mathbf{p}\right)}{\partial x} \frac{\partial f_{j}\left(r_{j} \mid \mathbf{p}\right)}{\partial x} \\
\frac{\partial f_{i}\left(r_{i} \mid \mathbf{p}\right)}{\partial y} \frac{\partial f_{i}\left(r_{i} \mid \mathbf{p}\right)}{\partial x} \frac{\partial f_{j}\left(r_{j} \mid \mathbf{p}\right)}{\partial y} \\
\frac{\partial f_{i}\left(r_{i} \mid \mathbf{p}\right)}{\partial y} \frac{\partial f_{j}\left(r_{i} \mid \mathbf{p}\right)}{\partial y}
\end{array}\right]\right\} .
\end{aligned}
$$

It easy to verify that for $i \neq j$, all terms in (34) are 0 so that:

$$
\begin{aligned}
\mathbf{J}(\mathbf{p})=\mathbb{E}_{\mathbf{r}} & \left\{\sum_{i=1}^{N_{\mathrm{r}}} \frac{1}{f_{i}\left(r_{i} \mid \mathbf{p}\right)^{2}}\right. \\
& \left.\times\left[\begin{array}{cc}
\left(\frac{\partial f_{i}\left(r_{i} \mid \mathbf{p}\right)}{\partial x}\right)^{2} & \frac{\partial f_{i}\left(r_{i} \mid \mathbf{p}\right)}{\partial x} \frac{\partial f_{i}\left(r_{i} \mid \mathbf{p}\right)}{\partial y} \\
\frac{\partial f_{i}\left(r_{i} \mid \mathbf{p}\right)}{\partial y} \frac{\partial f_{i}\left(r_{i} \mid \mathbf{p}\right)}{\partial x} & \left(\frac{\partial f_{i}\left(r_{i} \mid \mathbf{p}\right)}{\partial y}\right)^{2}
\end{array}\right]\right\} .
\end{aligned}
$$

The partial derivatives in (35) can be easily computed obtaining:

$$
\frac{\partial f_{i}\left(r_{i} \mid \mathbf{p}\right)}{\partial x}=f_{i}\left(r_{i} \mid \mathbf{p}\right)\left[D_{\mathrm{T}}\left(r_{i}\right) \frac{\partial}{\partial x} d_{\mathrm{T}}+D_{\mathrm{R} i}\left(r_{i}\right) \frac{\partial}{\partial x} d_{\mathrm{R} i}\right]
$$
and:

$$
\frac{\partial f_{i}\left(r_{i} \mid \mathbf{p}\right)}{\partial y}=f_{i}\left(r_{i} \mid \mathbf{p}\right)\left[D_{\mathrm{T}}\left(r_{i}\right) \frac{\partial}{\partial y} d_{\mathrm{T}}+D_{\mathrm{R} i}\left(r_{i}\right) \frac{\partial}{\partial y} d_{\mathrm{R} i}\right],
$$

where:

$$
D_{\mathrm{T}}\left(r_{i}\right)=\frac{-\alpha \sigma_{0}^{2}+\left(r_{i}-d_{\mathrm{T}}-d_{\mathrm{R} i}\right) d_{\mathrm{T}}^{-\alpha} d_{\mathrm{R} i}^{-\beta}\left(\alpha\left(r_{i}-d_{\mathrm{T}}-d_{\mathrm{R} i}\right)+2 d_{\mathrm{T}}\right)}{2 d_{\mathrm{T}}^{2} \sigma_{0}^{2}}
$$

$D_{\mathrm{R} i}\left(r_{i}\right)=\frac{-\beta \sigma_{0}^{2}+\left(r_{i}-d_{\mathrm{T}}-d_{\mathrm{R} i}\right) d_{\mathrm{T}}^{-\alpha} d_{\mathrm{R} i}^{-\beta}\left(\beta\left(r_{i}-d_{\mathrm{T}}-d_{\mathrm{R} i}\right)+2 d_{\mathrm{R} i}\right)}{2 d_{\mathrm{R} i}^{2} \sigma_{0}^{2}}$.

We have also:

$$
\begin{aligned}
\frac{\partial}{\partial x} d_{\mathrm{T}} & =\frac{x-x_{\mathrm{T}}}{d_{\mathrm{T}}}=\cos \phi \frac{\partial}{\partial y} d_{\mathrm{T}}=\frac{y-y_{\mathrm{T}}}{d_{\mathrm{T}}}=\sin \phi \\
\frac{\partial}{\partial x} d_{\mathrm{R} i} & =\frac{x-x_{\mathrm{R} i}}{d_{\mathrm{R} i}}=\cos \theta_{i} \frac{\partial}{\partial y} d_{\mathrm{R} i}=\frac{y-y_{\mathrm{R} i}}{d_{\mathrm{R} i}}=\sin \theta_{i}
\end{aligned}
$$

having indicated with $\phi$ the angle between the transmitter and the tag and with $\theta_{i}$ the angle between the $i$ th receiver and the tag both measured with respect to the horizontal, as illustrated in Fig. 4.

Considering the structure of the matrix $\mathbf{J}(\mathbf{p})$ in (35):

$$
\mathbf{J}(\mathbf{p})=\left[\begin{array}{ll}
J_{1,1}(\mathbf{p}) & J_{1,2}(\mathbf{p}) \\
J_{2,1}(\mathbf{p}) & J_{2,2}(\mathbf{p})
\end{array}\right]
$$

we obtain the terms:

$$
\begin{aligned}
J_{1,1}(\mathbf{p})= & \sum_{i=1}^{N_{\mathrm{r}}} \int_{-\infty}^{\infty} \frac{1}{\overline{f_{i}\left(r_{i} \mid \mathbf{p}\right)}\left(\frac{\partial f_{i}\left(r_{i} \mid \mathbf{p}\right)}{\partial x}\right)^{2} d r_{i}} \\
= & \sum_{i=1}^{N_{\mathrm{r}}} A_{i} \cos ^{2}(\phi)+B_{i} \cos ^{2}\left(\theta_{i}\right) \\
J_{2,2}(\mathbf{p})= & \sum_{i=1}^{N_{\mathrm{r}}} \int_{-\infty}^{\infty} \frac{1}{f_{i}\left(r_{i} \mid \mathbf{p}\right)}\left(\frac{\partial f_{i}\left(r_{i} \mid \mathbf{p}\right)}{\partial y}\right)^{2} d r_{i} \\
= & \sum_{i=1}^{N_{\mathrm{r}}} A_{i} \sin ^{2}(\phi)+B_{i} \sin ^{2}\left(\theta_{i}\right) \\
& +C_{i} \sin (\phi) \sin \left(\theta_{i}\right) \\
J_{1,2}(\mathbf{p})= & J_{2,1}(\mathbf{p})=\sum_{i=1}^{N_{\mathrm{r}}} \int_{-\infty}^{\infty} \frac{1}{f_{i}\left(r_{i} \mid \mathbf{p}\right)} \frac{\partial f_{i}\left(r_{i} \mid \mathbf{p}\right)}{\partial x} \frac{\partial f_{i}\left(r_{i} \mid \mathbf{p}\right)}{\partial y} d r_{i} \\
= & \sum_{i=1}^{N_{\mathrm{r}}} A_{i} \cos (\phi) \sin (\phi)+B_{i} \cos \left(\theta_{i}\right) \sin \left(\theta_{i}\right)
\end{aligned}
$$

with:

$$
\begin{aligned}
A_{i} & \triangleq \int_{-\infty}^{\infty} f_{i}\left(r_{i} \mid \mathbf{p}\right) D_{\mathrm{R} i}^{2}\left(r_{i}\right) d r_{i}=\frac{1}{\sigma_{0}^{2} d_{\mathrm{T}}^{\alpha} d_{\mathrm{R}}^{\beta}}+\frac{\beta^{2}}{2 d_{\mathrm{R} i}^{2}} \\
B_{i} & \triangleq \int_{-\infty}^{\infty} f_{i}\left(r_{i} \mid \mathbf{p}\right) D_{\mathrm{T}}^{2}\left(r_{i}\right) d r_{i}=\frac{1}{\sigma_{0}^{2} d_{\mathrm{T}}^{\alpha} d_{\mathrm{R}}^{\beta}}+\frac{\alpha^{2}}{2 d_{\mathrm{T}}^{2}} \\
C_{i} & \triangleq \int_{-\infty}^{\infty} f_{i}\left(r_{i} \mid \mathbf{p}\right) D_{\mathrm{T}}\left(r_{i}\right) D_{\mathrm{R} i}\left(r_{i}\right) d r_{i} \\
& =\frac{2}{\sigma_{0}^{2} d_{\mathrm{T}}^{\alpha} d_{\mathrm{R}}^{\beta}}+\frac{\alpha \beta}{d_{\mathrm{T}} d_{\mathrm{R} i}} .
\end{aligned}
$$

Finally, from the previous equations, we can compute the PEB as:

$$
\operatorname{PEB}(\mathbf{p})=\sqrt{\frac{J_{1,1}(\mathbf{p})+J_{2,2}(\mathbf{p})}{\operatorname{det} \mathbf{J}(\mathbf{p})}} .
$$

Result (48), with (42)-(47), provides a closed form of the attainable performance of any position estimator as a function of network topology and tag position, as well 


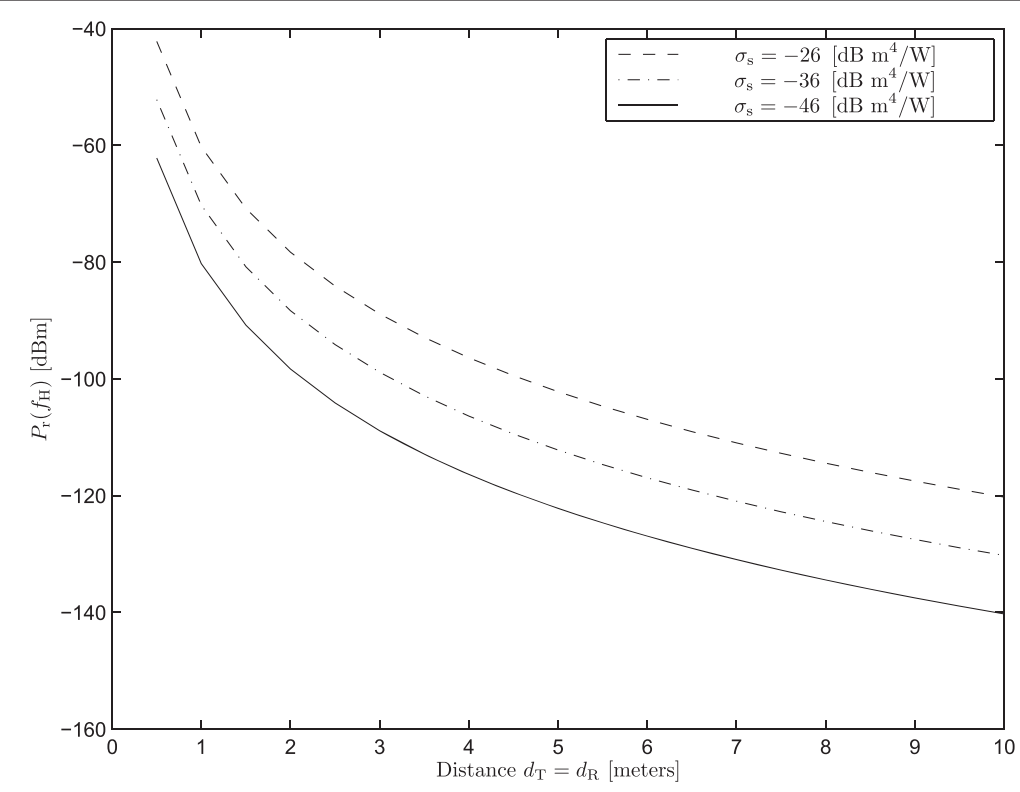

Fig. $5 P_{r}\left(f_{H}\right)(\mathrm{dBm})$ as a function of the tag distance $d_{T}=d_{R}$

as ranges estimate quality, and represents a useful benchmark for any practical estimator. Specifically, such a theoretical performance gives the asymptotic performance of maximum likelihood (ML) estimators achievable for large SNRs or long observation times [36].

\section{Numerical results}

We present some numerical results with the purpose to show the performance limits in terms of detection and ranging/localization accuracy of harmonic tags in a typical UHF RFID context. The following system parameters are considered: $f_{1}=865 \mathrm{MHz}, f_{2}=867 \mathrm{MHz}$, EIRP $=30 \mathrm{dBm}, G_{\mathrm{rx}}\left(f_{\mathrm{H}}\right)=5 \mathrm{~dB}$, PLL $B_{\mathrm{eq}}=100 \mathrm{~Hz}$, and receiver noise figure $F=5 \mathrm{~dB}$. The harmonic tag configuration and parameters are that described in the first section.

First, we consider a scenario in which the transmitter and receiver are co-located and the tag is positioned at distance $d_{\mathrm{T}}=d_{\mathrm{R}}$. Figures 5 and 6 show, respectively,

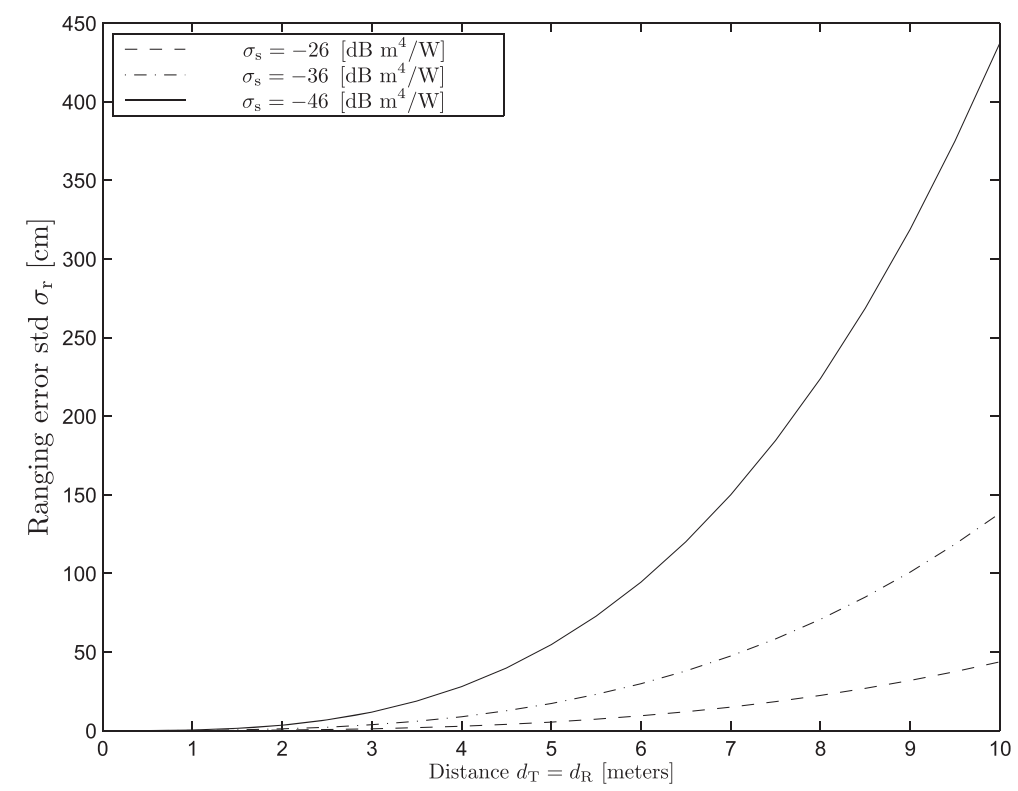

Fig. 6 Ranging error standard deviation as a function of the tag distance $d_{T}=d_{R}$ 


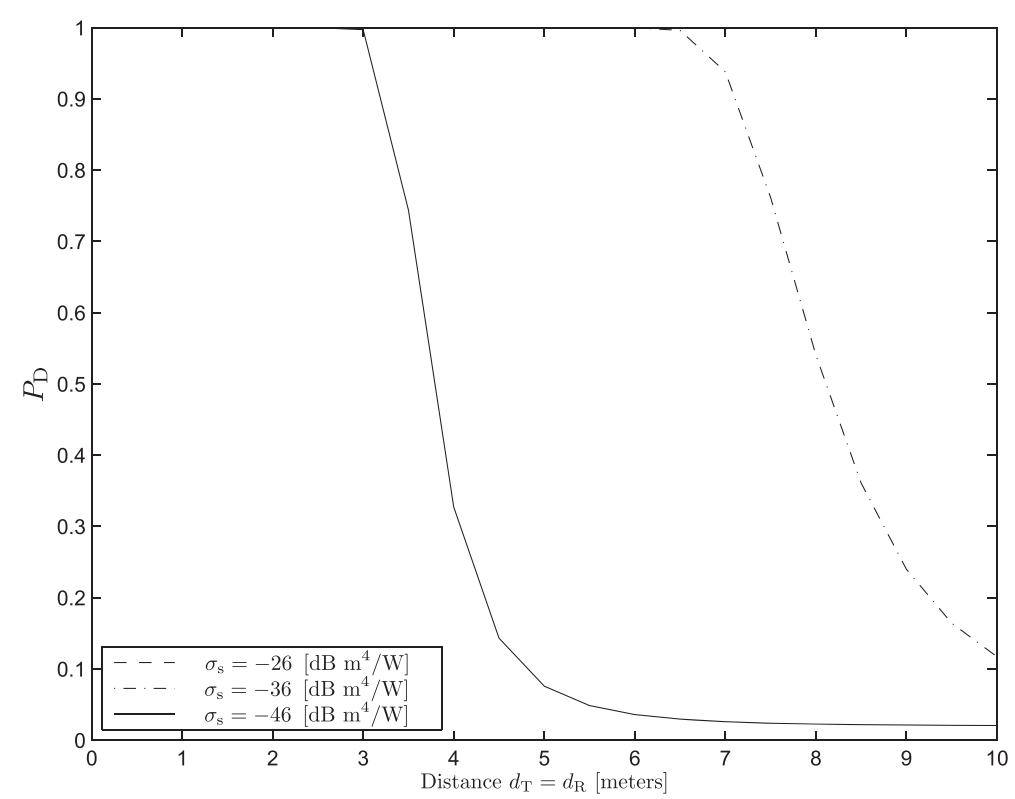

Fig. 7 Probability of detection as a function of the tag distance $d_{T}=d_{R} \cdot P_{\mathrm{F}}=10^{-4}$

the received power $\operatorname{Pr}\left(f_{\mathrm{H}}\right)$ of the received harmonic components at $2 f_{1}$ and $2 f_{2}$ and the ranging error standard deviation $\sigma_{\mathrm{r}}$ for increasing tag distances and for different values of the SHRCS $\sigma_{\mathrm{s}}$. As can be noted, also considering a penalty of $20 \mathrm{~dB}$ with respect to the optimum tag configuration analyzed in the first section, corresponding to $\sigma_{\mathrm{s}}=-26\left[\mathrm{~dB} \mathrm{~m}{ }^{4} / \mathrm{W}\right]$, the ranging accuracy stays below $40 \mathrm{~cm}$ within a range of $4 \mathrm{~m}$. The best configuration leads to the same accuracy up to $10 \mathrm{~m}$. The probability of tag detection $P_{\mathrm{D}}$ is reported in Fig. 7 for the same configurations having fixed a target false alarm probability $P_{\mathrm{F}}=$ $10^{-4}$. The curve corresponding to $\sigma_{\mathrm{s}}=-26\left[\mathrm{~dB} \mathrm{~m}^{4} / \mathrm{W}\right]$ is constant to 1 for the operating ranges considered, whereas the tag is detectable ( $P_{\mathrm{D}}$ close to 1$)$ up to 3 and $6 \mathrm{~m}$ for $\sigma_{\mathrm{s}}=-36\left[\mathrm{~dB} \mathrm{~m}^{4} / \mathrm{W}\right]$ and $\sigma_{\mathrm{s}}=-46\left[\mathrm{~dB} \mathrm{~m}^{4} / \mathrm{W}\right]$, respectively.

Under a practical implementation perspective of the proposed scheme, when the transmitter and receiver are

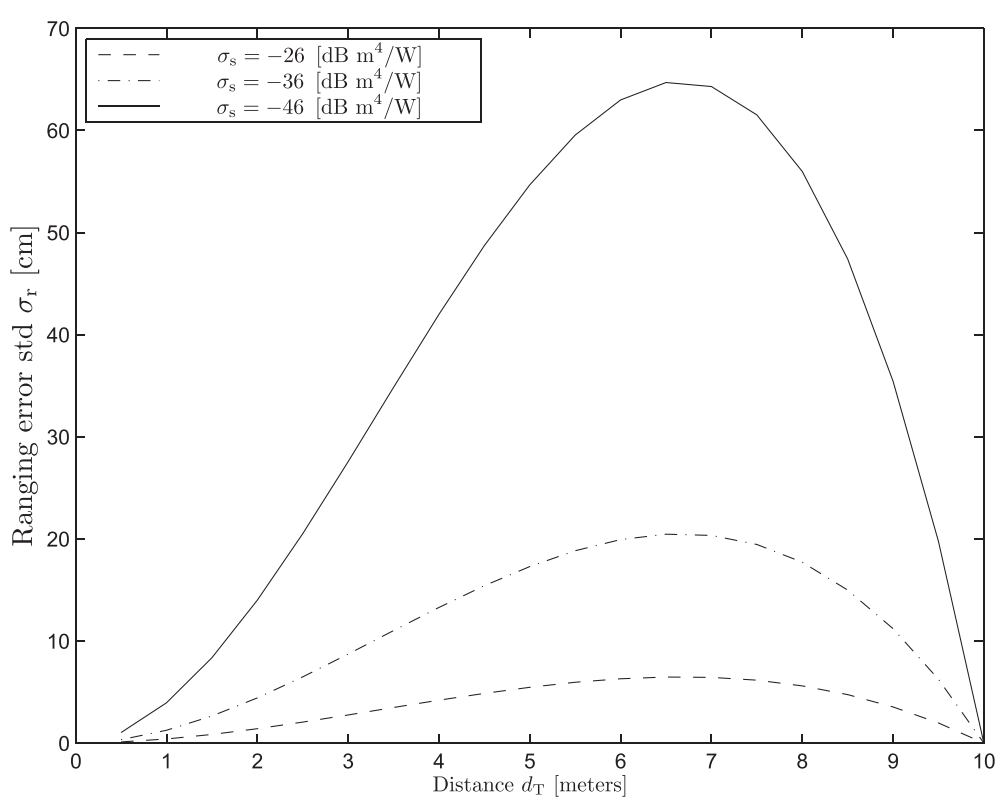

Fig. 8 Ranging error standard deviation as a function of the distance $d_{T}$ with a fixed receiver at $10 \mathrm{~m}$ from the transmitter 


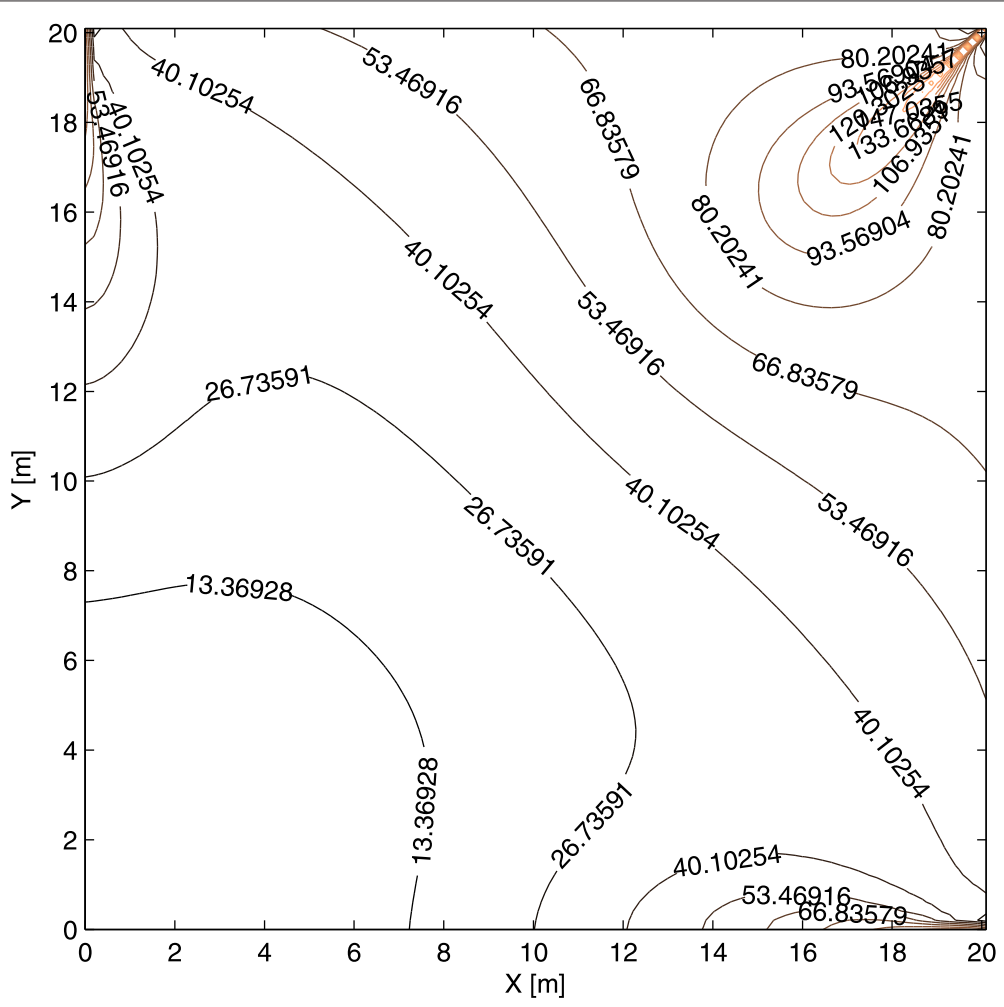

Fig. 9 Position error bound PEB $(\mathbf{p})[\mathrm{cm}]$ with four receivers at the corners and one transmitter located at $(0,0) .20 \times 20 \mathrm{~m}$ area. $\sigma_{\mathrm{s}}=-26\left[\mathrm{~dB} \mathrm{~m}^{4} / \mathrm{W}\right]$

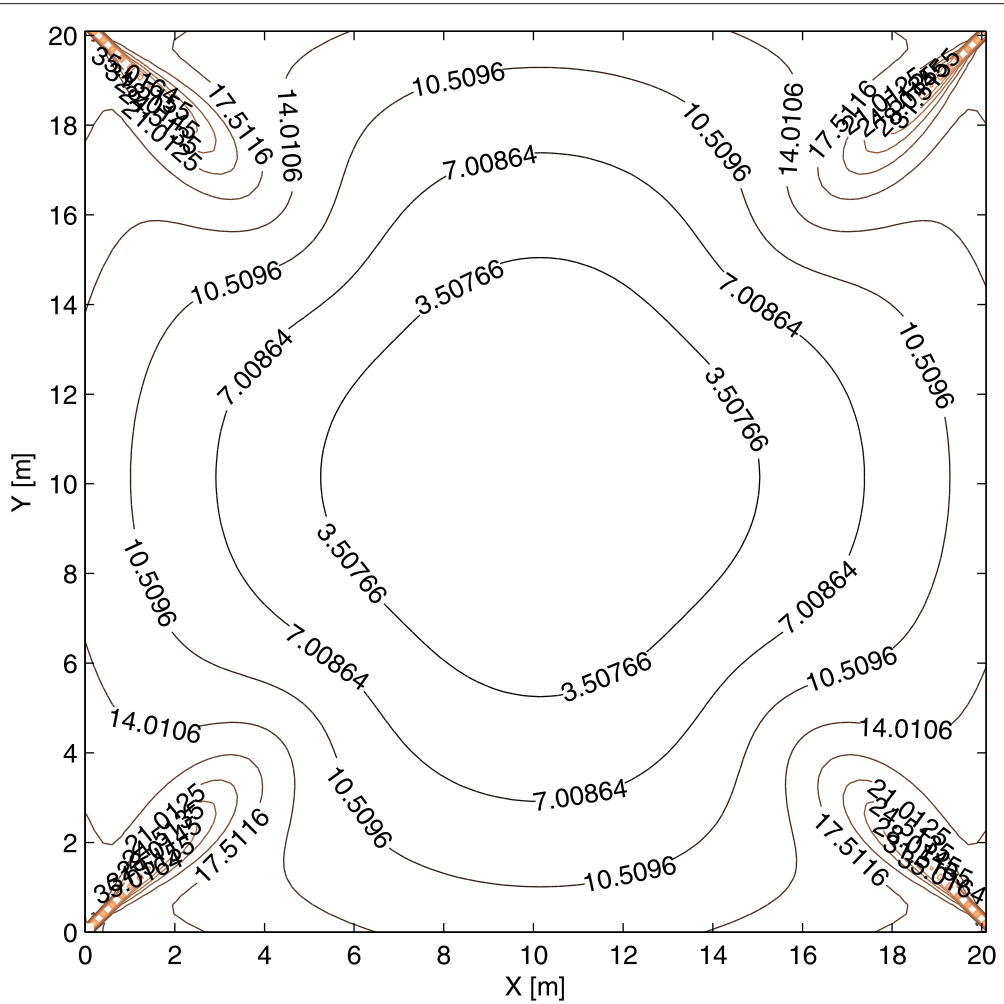

Fig. 10 Position error bound PEB $(\mathbf{p})[\mathrm{cm}]$ with four receivers at the corners and one transmitter located in the center $(10,10) .20 \times 20 \mathrm{~m}$ area. $\sigma_{\mathrm{s}}=-26\left[\mathrm{~dB} \mathrm{~m} \mathrm{~m}^{4} / \mathrm{W}\right]$ 
co-located, a second harmonic component caused by the distortion of the transmitter's power amplifier might arise and compromise the detection capability of the receiver. As a consequence, such an unwanted coupling component has to be properly reduced, for example, by inserting a filter after the power amplifier. To have an idea of the required rejection capability of the filter, let us consider the following numerical example: Assume a typical harmonic spurious at UHF not larger than $-60 \mathrm{dBc}$, which means about $-30 \mathrm{dBm}$ harmonic emission power with a transmitter power of $30 \mathrm{dBm}$. Considering a SNR of at least $10 \mathrm{~dB}$ for tag detectability and a receiver sensitivity of about $-120 \mathrm{dBm}$ (see Fig. 5) at typical distance of 7-9 m, the maximum tolerable power leakage is about $-130 \mathrm{dBm}$ resulting in a filter rejection of $100 \mathrm{~dB}$, which can be easily implemented considering that the harmonic is spaced of more than $800 \mathrm{MHz}$ with respect to the fundamental. Note that in a multistatic scenario, like that investigated in the following, the power amplifier distortion does not represent an issue, as the TX and RX are located in different positions.

A different layout is investigated in Fig. 8 where the receiver is located $10 \mathrm{~m}$ away from the transmitter and the tag is positioned at a distance $d_{\mathrm{T}}$ from the transmitter and $d_{\mathrm{R}}=10-d_{\mathrm{T}} \mathrm{m}$ from the receiver along the line connecting the transmitter and receiver. The different impact of $d_{\mathrm{T}}$ with respect to $d_{\mathrm{R}}$ in (17), which is a consequence of the dependence of the harmonic RCS on the impinging power, leads to an asymmetric behavior where the worst situation does not correspond to the middle distance $d_{\mathrm{T}}=d_{\mathrm{R}}=5 \mathrm{~m}$. Specifically, it can be shown that it is better to keep the tag as much as possible close to the transmitter. In general layouts, employing the transmitter close to the tag is preferable with respect to layouts in which the receiver is close but the transmitter is far.

Finally, we analyze a more complex scenario employing one transmitter and $N_{\mathrm{r}}=4$ receivers located at the corners of a square area of $20 \times 20 \mathrm{~m}$. Figure 9 shows the contour plot of the PEB in (48) related to a harmonic tag located in the area when the transmitter is placed in one corner. As can be noticed, an accuracy of $10-20 \mathrm{~cm}$ can be achieved only in those spatial configurations close to the transmitter. A different result is obtained by placing the transmitter in the center of the area, as shown in Fig. 10. In fact, now an accuracy better than $15 \mathrm{~cm}$ is achieved for most of the locations in the area thus remarking the opportunity to prefer layouts with a central transmitter deployment.

By comparison, consider the use of FM chirp signals like in [22] where, to provide a sufficient ranging accuracy, signals with a bandwidth of $B=20 \mathrm{MHz}$ are considered. ${ }^{7}$ Note that such large bandwidth leads to a noise at the receiver which is $B / B_{\mathrm{eq}}=50 \mathrm{~dB}$ higher than that present in our scheme. This means that, under the same setting, a penalty of about $50 \mathrm{~dB}$ in terms of SNR is paid using FM chirp signals that has to be compensated by employing high-directivity antennas and high-power levels $(>1 \mathrm{KW})$, as done in [22]. With our solution, no mechanical-steered high-directional antennas are needed thus facilitating the localization of the tag using multiple receivers and relative low-power levels ( $30 \mathrm{dBm}$ EIRP).

\section{Conclusions}

In this paper, we investigated, from the theoretical point of view, the detection and ranging capabilities of harmonic tags by proposing a simple two-tone interrogation signal and a dedicated receiver scheme. We have introduced the specific harmonic radar cross section (SHRCS) concept to describe concisely the intensity of the backscattered harmonic components of the tag that depends on the power density impinging the tag's antenna. The analysis has been successively extended to include the presence of more receivers to provide accurate tag localization. A closed form of the attainable localization accuracy has been derived and provides a useful tool for system design and layout optimization. Results show that very accurate tag detection and localization in the order of few centimeters are possible in a typical area of $10-20 \mathrm{~m}$ using the RFID UHF band.

\section{Endnotes}

${ }^{1} Z_{\mathrm{d}}\left(f_{\mathrm{L}}\right)$ could also account for the impedance of the matching network eventually included in the tag to maximize the power transfer to the diode [33].

${ }^{2}$ For simplicity, we do not consider the angular dependency of the RCS although it can be easily accounted for in the analysis.

${ }^{3}$ For instance, a straightforward method to generate coherent tones at $f_{1}$ and $f_{2}$ is to start from a baseband oscillation at frequency $\Delta f / 2$ and use it as input of an up-converter with carrier frequency $f_{0}=\left(f_{1}+f_{2}\right) / 2$.

${ }^{4}$ Operator $\mathbb{E}[\cdot]$ denotes the statistical expectation.

${ }^{5} A \succeq B$ means that $A-B$ is a non-negative definite.

${ }^{6}$ For simplicity of notation, we do not indicate explicitly the dependence of $d_{\mathrm{T}}$ and $d_{\mathrm{R} i}$ on $\mathbf{p}$.

${ }^{7}$ In fact, the ranging resolution of FM chirp radars is about $c / 2 B$.

Competing interests

The author declares that he has no competing interests.

\section{Acknowledgements}

The author would like to thank Gianni Pasolini, Francesco Guidi, Nicoló Decarli, and Anna Guerra for the useful discussions.

This research was supported by the Italian Minister of Research through the GRETA project (grant no. 2010WHY5PR).

Received: 6 February 2015 Accepted: 6 August 2015

Published online: 20 August 2015 


\section{References}

1. K Finkenzeller, RFID Handbook: Fundamentals and Applications in Contactless Smart Cards and Identification. Second Edition. (Wiley, New York, 2004)

2. V Chawla, DS Ha, An overview of passive RFID. IEEE Commun. Mag. 45(9), 11-17 (2007)

3. A Costanzo, D Masotti, T Ussmueller, R Weigel, Tag, you're it: ranging and finding via RFID technology. IEEE Microw. Mag. 14(5), 36-46 (2013)

4. G Marrocco, L Mattioni, C Calabrese, Multiport sensor RFIDs for wireless passive sensing of objects - basic theory and early results. IEEE Trans. Antennas Propag. 56(8), 2691-2702 (2008)

5. PV Nikitin, R Martinez, S Ramamurthy, H Leland, G Spiess, KVS Rao, in 2010 IEEE International Conference on RFID. Phase based spatial identification of UHF RFID tags, (2010), pp. 102-109. doi:10.1109/RFID.2010.5467253

6. D Dardari, R D'Errico, C Roblin, A Sibille, MZ Win, Ultrawide bandwidth RFID: the next generation?. Proc. IEEE. 98(9), 1570-1582 (2010)

7. D Dardari, F Guidi, C Roblin, A Sibille, Ultra-wide bandwidth backscatter modulation: processing schemes and performance. EURASIP J. Wirel. Commun. Netw. 47(1), 1-15 (2011)

8. N Decarli, F Guidi, D Dardari, A novel joint RFID and radar sensor network for passive localization: design and performance bounds. IEEE J. Sel. Topics Signal Process. 8(1), 80-95 (2014). doi:10.1109/JSTSP.2013.2287174. Special Issue on Non-cooperative Localization Networks

9. S Preradovic, NC Karmakar, Chipless RFID: bar code of the future. IEEE Microw. Mag. 11(7), 87-97 (2010)

10. GL Charvat, EJ Rothwell, LC Kempel, in 2003 IEEE Antennas and Propagation Society International Symposium. Harmonic radar tag measurement and characterization, vol. 2, (2003), pp. 696-6992. doi:10.1109/APS.2003.1219331

11. S Kim, C Mariotti, F Alimenti, P Mezzanotte, A Georgiadis, A Collado, L Roselli, MM Tentzeris, No battery required: perpetual RFID-enabled wireless sensors for cognitive intelligence applications. IEEE Microw. Mag. 14(5), 66-77 (2013)

12. RECCO Rescue System, A system for locating avalanche victims, (Recco AB, Lidingo, Sweden. [Online] Available: http://www.recco.com/about

13. C Li, VM Lubecke, O Boric-Lubecke, J Lin, A review on recent advances in Doppler radar sensors for noncontact healthcare monitoring. IEEE Trans. Microw. Theory Tech. 61(5), 2046-2060 (2013). doi:10.1109/TMTT. 2013.2256924

14. L Chioukh, H Boutayeb, K Wu, D Deslandes, in 2011 European Radar Conference (EURAD). Monitoring vital signs using remote harmonic radar concept IEEE Manchester (UK), 2011), pp. 381-384

15. A Singh, V Lubecke, in 2011 IEEE Topical Conference on Biomedical Wireless Technologies, Networks, and Sensing Systems (BioWireleSS). Body-worn passive planar harmonic tag design for use with Doppler radar, (2011), pp. 51-54. doi:10.1109/BIOWIRELESS.2011.5724358

16. L Cabria, JA Garcia, E Malaver, A Tazon, A PHEMT frequency doubling active antenna with BPSK modulation capability. IEEE Antennas Wireless Propag. Lett. 3(1), 310-313 (2004). doi:10.1109/LAWP.2004.838821

17. T Aballo, L Cabria, JA Garcia, T Fernandez, F Marante, in 36th European Microwave Conference 2006. Taking advantage of a Schottky junction nonlinear characteristic for radiofrequency temperature sensing, (2006), pp. 318-321. doi:10.1109/EUMC.2006.281320

18. IT Nassar, TM Weller, JL Frolik, A compact 3-D harmonic repeater for passive wireless sensing. IEEE Trans. Microw. Theory Tech. 60(10), 3309-3316 (2012). doi:10.1109/TMTT.2012.2210440

19. F Alimenti, L Roselli, Theory of zero-power RFID sensors based on harmonic generation and orthogonally polarized antennas. Prog. Electromagn. Res. 134, 337-357 (2013)

20. D Mascanzoni, H Wallin, The harmonic radar: a new method of tracing insects in the field. Ecol. Entomol. 11, 387-390 (1986)

21. H Aumann, E Kus, B Cline, NW Emanetoglu, in 2013 IEEE International Instrumentation and Measurement Technology Conference (I2MTC). A low-cost harmonic radar for tracking very small tagged amphibians (2013), pp. 234-237. doi:10.1109/I2MTC.2013.6555415

22. BG Colpitts, G Boiteau, Harmonic radar transceiver design: miniature tags for insect tracking. IEEE Trans. Antennas Propag. 52(11), 2825-2832 (2004). doi:10.1109/TAP.2004.835166

23. JR Riley, AD Smith, Design considerations for an harmonic radar to investigate the flight of insects at low altitude. Comput. Electron. Agric. 35(2-3), 151-169 (2002). doi:10.1016/S0168-1699(02)00016-9
24. RD Brazee, ES Miller, ME Reding, MG Klein, B Nudd, H Zhu, A transponder for harmonic radar tracking of the black vine weevil in behavioral research. Trans. Am. Soc. Agric. Eng. 38(2), 831-838 (2005)

25. C Fazi, F Crowne, M Ressler, Design considerations for nonlinear scattering. Army research lab Adelphi MD sensors and electron devices directorate - ADA549867, 1-9 (2011)

26. A Singh, V Lubecke, in 2012 IEEE MTT-S International Microwave Symposium Digest (MTT). Adaptive noise cancellation for two frequency radars using frequency doubling passive RF tags, (2012), pp. 1-3. doi:10.1109/ MWSYM.2012.6259694

27. F Crowne, C Fazi, in International Radar Conference-Surveillance for a Safer World, 2009 (RADAR). Nonlinear radar signatures from metal surfaces (IEEE Bordeaux (France), 2009), pp. 1-6

28. C Mikeka, H Arai, in Proceedings of the 5th European Conference on Antennas and Propagation (EUCAP). Dual-band RF energy-harvesting circuit for range enhancement in passive tags (IEEE Rome (Italy), 2011), pp. 1210-1214

29. PV Nikitin, KVS Rao, in IEEE Antennas and Propagation Society International Symposium, 2009 (APSURSI '09). Harmonic scattering from passive UHF RFID tags, (2009), pp. 1-4. doi:10.1109/APS.2009.5171788

30. JD McCann, Detection system. Patent US4,303,910 (1981)

31. BE Jones, JA Smith, Transponders. Patent US 4,160,971 (1979)

32. M Kanda, Analytical and numerical techniques for analyzing electrically short dipole with a nonlinear load. IEEE Trans. Antennas Propag. 28(1) 71-78 (1980)

33. H Aumann, E Kus, B Cline, NW Emanetoglu, in Proceedings of the 2012 IEEE Antennas and Propagation Society International Symposium (APSURSI). An asymmetrical dipole tag with optimum harmonic conversion efficiency (Chicago (IL), 2012), pp. 1-2

34. HSMS-286Y microwave Schottky detector diodes in surface mount SOD-523 package. AVAGO Technologies - Data Sheet (2006)

35. CA Balanis, Antenna Theory, 2nd edn. (John Wiley \& Sons, Inc, New York, 1997)

36. HLV Trees, Detection, Estimation, and Modulation Theory: Part I, 2nd edn. (John Wiley \& Sons, Inc., New York, 2001)

37. JG Proakis, Digital Communications, 4th edn. (McGraw-Hill, Inc., New York, 2001)

38. JD Parsons, The Mobile Radio Propagation Channel, 2nd edn. (John Wiley \& Sons, Inc., New York, 2000)

39. D Jourdan, D Dardari, MZ Win, Position error bound for UWB localization in dense cluttered environments. IEEE Trans. Aerosp. Electron. Syst. 44(2), 613-628 (2008)

\section{Submit your manuscript to a SpringerOpen ${ }^{\circ}$ journal and benefit from:}

- Convenient online submission

Rigorous peer review

- Immediate publication on acceptance

- Open access: articles freely available online

- High visibility within the field

- Retaining the copyright to your article

Submit your next manuscript at $>$ springeropen.com 